\title{
Mediterranean plant karyological data - 28
}

\author{
edited by G. Kamari, C. Blanché \& S. Siljak-Yakovlev
}

\begin{abstract}
Kamari, G., Blanché, C. \& Siljak-Yakovlev, S. (eds): Mediterranean plant karyological data 28. - Fl. Medit. 28: 419-445. 2018. — ISSN: 1120-4052 printed, 2240-4538 online.

This is the twenty-eight of a series of karyological data from Mediterranean area, peri-Alpine communities and the Atlantic Islands, in English or French language. It comprises contributions on 27 taxa: Alnus, Erythronium, Genista, Leucanthemum, Melittis, Orchis, Stachys, Scorzonera from Bosnia-Herzegovina and F.Y.R.O.M. by F. Pustahija, N. Bašić \& S. Siljak-Yakovlev (Nos 19351942); Taraxacum from Turkey by B. Gürdal \& N. Özhatay (Nos 1943-1954); Centaurea, Cichorium, Glebionis, Pallenis, Lactuca, Phagnalon, Silybum from Morocco by A. Gounssa, K. Hormat, A. El Aboudi, F. Ezzahra, F. El Alaoui Faris \& M. Arahou (Nos 1955-1961).
\end{abstract}

Addresses of the editors:

Prof. Emer. Georgia Kamari, Botanical Institute, Department of Biology, University of Patras, GR26500 Patras, Greece. E-mail: kamari@upatras.gr

Prof. Cesar Blanché, IRBio-GReB, Laboratori de Botànica, Facultat de Farmàcia, Universitat de Barcelona, Av. Joan XXIII s/n, E-08028 Barcelona, Catalonia, Spain. E-mail: cesarblanche@ub.edu

Dr. Sonja Siljak-Yakovlev, Ecologie, Systématique et Evolution, Département "Evolution des angiospermes", Univ. Paris-Sud, CNRS, AgroParisTech, Université Paris-Saclay, Bât 360, 91405 Orsay cedex, France. E-mail: sonia.yakovlev@u-psud.fr 